\title{
A Reduction Technique for RLCG Interconnects Using Least Squares Method
}

\author{
Junji Kawata $^{1}$, Yuichi Tanji ${ }^{2}$, Yoshifumi Nishio ${ }^{3}$ and Akio Ushida ${ }^{1}$ \\ 1 Tokushima Bunri University, \\ 1314-1 Shido, Sanuki-shi, Japan \\ 2 Kagawa University, \\ 3 Tokushima University, \\ \{kawata,ushida\}@fe.bunri-u.ac.jp \\ Phone/FAX:+81-87-894-5111/+81-87-894-4201 \\ tanji@eng.kagawa-u.ac.jp \\ nishio@ee.tokushima-u.ac.jp
}

\section{Introduction}

The analysis and design of high speed LSI chips are becoming more and more important, because the sub-circuits coupled with interconnects embedded in the substrate sometimes cause the fault switching operations due to the signal delays, crosstalks, reflections and so on [1]-[5]. The Elmore resistance-capacitance (RC) delay metric is popular due to its simple closed-form expression, computation speed and fidelity with respect to the simulation [4]. The closed-form combining with the delay and crosstalk is firstly presented in the reference [5]. The modified algorithms are proposed later for the improvement of the accuracy and the practical applications in the simulations [6]-[9].

Nowadays, AWE (asymptotic waveform evaluation method) [10] is widely used as a reduction technique of the large scale networks coupled with interconnects, the algorithm is based on a moment-matching technique and Padé approximation. However, the method sometimes become erroneous, if there exist the poles far from the origin. To overcome the problem, CFH (complex frequency hopping) [11] and multi-point Padé approximation [12] methods are proposed. In these reduction algorithms, the reduced circuits sometimes become unstable in the time domain even if all the poles are located in the left hand side of the complex plane. The ill-condition can be overcome by PVL (Padé via Lanczos process) [13], and PRIMA (passive reduced-order interconnect macromodeling algorithm) [2,14]. In order to apply these algorithms to the interconnects, we need two steps such that each interconnect is firstly modeled by a finite order system, and Arnoldi-based congruence transformation is applied to the system to form its reduced order model.

In this paper, we consider LSIs such as ASIC or SoC (System on a Chip) are coupled with interconnects embedded in the substrate. In this case, the diffusion resistance components of the interconnects are generally assumed to be very large compared to those of PCBs [3] and the lengths are very short. From the telegraph equation to interconnect, The admittance matrices can be derived from the relations at the near and far ends [1]. We propose here a new computational algorithm for calculating the exact poles in the complex plane. Next, each of the elements of the admittance matrices is approximated by partial fraction using a few dominant poles around the origin, where the corresponding residues are evaluated by either a theoretical method or the least squares method. The latter method will be useful for relatively large scale multi-conductor interconnects. Thus, the admittance matrices are reduced as the partial fractions by which it is possible to derive asymptotic equivalent circuits. We show the numerical methods for calculating the poles and residues in section 2. Illustrative examples are given in section 3 .

\section{Calculation of exact poles and residues}

Now, consider a uniform $N$ coupled RLCG interconnect described by the following telegraph equations;

$$
\left.\begin{array}{l}
\frac{d \mathbf{V}(x, s)}{d x}=-(\mathbf{R}+s \mathbf{L}) \mathbf{I}(x, s) \\
\frac{d \mathbf{I}(x, s)}{d x}=-(\mathbf{G}+s \mathbf{C}) \mathbf{V}(x, s)
\end{array}\right\}
$$

The input and output relations at the near and far ends are described by the admittance matrices as follows [1]:

$$
\left[\begin{array}{r}
\mathbf{I}(0, s) \\
-\mathbf{I}(d, s)
\end{array}\right]=\left[\begin{array}{ll}
\mathbf{Y}_{11}(s) & \mathbf{Y}_{12}(s) \\
\mathbf{Y}_{21}(s) & \mathbf{Y}_{22}(s)
\end{array}\right]\left[\begin{array}{c}
\mathbf{V}(0, s) \\
\mathbf{V}(d, s)
\end{array}\right]
$$

where, using the eigenvalues $\boldsymbol{\Gamma}(s)=\operatorname{diag}\left[\gamma_{i}(s)\right]$, we have the following relations;

$$
\left.\begin{array}{rl}
\mathbf{Y}_{11}(s) & =\mathbf{Y}_{22}(s)=\mathbf{P}_{c}(s) \operatorname{diag}\left[\operatorname{coth} \gamma_{i}(s) d\right] \mathbf{P}_{v}(s)^{-1} \\
\mathbf{Y}_{12}(s) & =\mathbf{Y}_{21}(s)=\mathbf{P}_{c}(s) \operatorname{diag}\left[\sinh \gamma_{i}(s) d\right]^{-1} \mathbf{P}_{v}(s)^{-1}
\end{array}\right\}
$$

where

$$
\left.\begin{array}{l}
\operatorname{diag}\left[\gamma_{i}(s)^{2}\right]=\mathbf{P}_{v}(s)^{-1}(\mathbf{R}+s \mathbf{L})(\mathbf{G}+s \mathbf{C}) \mathbf{P}_{v}(s) \\
\operatorname{diag}\left[\gamma_{i}(s)^{2}\right]=\mathbf{P}_{c}(s)^{-1}(\mathbf{G}+s \mathbf{C})(\mathbf{R}+s \mathbf{L}) \mathbf{P}_{c}(s)
\end{array}\right\}
$$

Thus, we have

$$
\begin{gathered}
\mathbf{P}_{c}(s)=(\mathbf{R}+s \mathbf{L})^{-1} \mathbf{P}_{v}(s) \boldsymbol{\Gamma}(s) \\
\mathbf{P}_{c}^{T}(s)=\mathbf{P}_{v}(s)^{-1}
\end{gathered}
$$


Observe that all the poles of admittance matrices can be found at the locations satisfying $\sinh \gamma_{i}(s)=0, i=1,2, \ldots, N$. Thus, we have the following theorem for calculations of the exact poles.

Theorem 1: The locations of poles satisfying relations (3) are found by solving the following equation:

$$
\left|(\mathbf{R}+s \mathbf{L})(\mathbf{G}+s \mathbf{C})+\left(\frac{n \pi}{d}\right)^{2} \mathbf{I}\right|=0, n=1,2, \ldots
$$

where $d$ is the length of interconnect.

Proof: In the case of $n \neq 0$, we have from (3) that the poles satisfy the following relation:

$$
\left|\mathbf{P}_{v}(s) \operatorname{diag}\left[\sinh \gamma_{i}(s) d\right] \mathbf{P}_{c}(s)^{-1}\right|=0
$$

and

$$
\left|\mathbf{P}_{v}(s) \operatorname{diag}\left[\tanh \gamma_{i}(s) d\right] \mathbf{P}_{c}(s)^{-1}\right|=0
$$

Since the transfer matrices $\mathbf{P}_{v}(s)$ and $\mathbf{P}_{c}(s)$ are nonsingular for the nonzero eigenvalues, the poles satisfying the above two relations are given by

$$
\gamma_{i}(s) d=j n \pi, \quad i=1,2, \ldots, N, \quad n=1,2, \ldots
$$

Therefore, the characteristic equation from the telegraph equation (1) has to satisfy the relation (5).

\section{Q.E.D.}

Corollary 1.1: The poles at $n=0$ satisfy the following relation;

$$
|\mathbf{R}+s \mathbf{L}|=0
$$

Proof: We have $\gamma_{i}=0$ at $n=0$. Thus, using the relations of (4), we have the following relation;

$$
\begin{aligned}
& \lim _{\gamma_{i} \rightarrow 0} \mathbf{P}_{c}(s) \operatorname{diag}\left[\sinh \gamma_{i}(s) d\right]^{-1} \mathbf{P}_{v}(s)^{-1} \\
& =(\mathbf{R}+s \mathbf{L})^{-1} \mathbf{P}_{v}(s) \operatorname{diag}\left[\gamma_{i}(s)\right] \frac{1}{\operatorname{diag}\left[\gamma_{i}(s) d\right]} \mathbf{P}_{v}(s)^{-1} \\
& =\frac{1}{d}(\mathbf{R}+s \mathbf{L})^{-1}
\end{aligned}
$$

\section{Q.E.D.}

In generally, it is not easy to calculate the poles using the relation (5). Hence, we apply Leverrier-Faddeeva algorithm [16] for getting the characteristic equation of $|s \mathbf{I}-\mathbf{A}|=0$ to the relation (5). We set in (5)

$$
\mathbf{A}(s)=-(\mathbf{R C}+\mathbf{L G})^{-1}\left[\mathbf{R G}+\left(\frac{n \pi}{d}\right)^{2} \mathbf{I}+s^{2} \mathbf{L} \mathbf{C}\right]
$$

Then, we have

$|s \mathbf{I}-\mathbf{A}(s)|=\alpha_{0}(s)+s \alpha_{1}(s)+\cdots+s^{N-1} \alpha_{N-1}(s)+s^{N}$ where $\alpha_{k}(s)$ is given by the following relations:

$$
\begin{aligned}
& \mathbf{B}_{N-1}=\mathbf{I} \\
& \quad \Rightarrow \quad \alpha_{N-1}(s)=-\operatorname{tr}(\mathbf{A}(s)) \\
& \mathbf{B}_{N-2}(s)=\mathbf{A}(s) \mathbf{B}_{N-1}+\alpha_{N-1}(s) \mathbf{I} \\
& \quad \Rightarrow \quad \alpha_{N-2}(s)=-\frac{1}{2} \operatorname{tr}\left(\mathbf{B}_{N-2}(s) \mathbf{A}(s)\right) \\
& \quad \mathbf{B}_{0}(s)=\mathbf{A}(s) \mathbf{B}_{1}(s)+\alpha_{1}(s) \mathbf{I} \\
& \quad \Rightarrow \quad \alpha_{0}(s)=-\frac{1}{N} \operatorname{tr}\left(\mathbf{B}_{0}(s) \mathbf{A}(s)\right)
\end{aligned}
$$

Note that the maximum degree of (11) eventually becomes $2 N$, because $\alpha_{k}(s)$ is polynomial with $s$. This algebraic equation can be numerically solved by the use of Bairstow method and so on. In this way, we can calculate the exact poles of the admittance matrices (3).

Then the corresponding residues are calculated by either the least squares method or a theoretical method. The detail of the theoretical method can be found in [17].

We choose the dominant poles located around the origin of complex plane, because such poles will have a great effect on the transient response. On the contrary, the poles stay away from the origin will give only small effect. Thus, the admittance matrices given by (3) are approximated by the partial fractions, which consist of the dominant poles and the corresponding residues, in the following form:

$$
\begin{aligned}
& Y_{11, i j}(s)=Y_{22, i j}(s)= \\
& \frac{k_{1, i j}}{s+p_{0}}+\sum_{k=1}^{M} \frac{s k_{C 1, k, 1, i j}+k_{C 0, k, 1, i j}}{s^{2}+a_{C, k} s+b_{C, k}} \\
& Y_{12, i j}(s)=Y_{21, i j}(s)= \\
& \frac{k_{2, i j}}{s+p_{0}}+\sum_{k=1}^{M} \frac{s k_{C 1, k, 2, i j}+k_{C 0, k, 2, i j}}{s^{2}+a_{C, k} s+b_{C, k}}
\end{aligned}
$$

where $M$ shows the number of complex conjugate pairs. As shown in [17], the absolute values of $k_{2, i j}, k_{C 1, k, 2, i j}$ and $k_{C 0, k, 2, i j}$ are equal to $k_{1, i j}, k_{C 1, k, 1, i j}$ and $k_{C 0, k, 1, i j}$, respectively, but they alternately contain negative sign. Therefore, the relation (2) can be written as follows;

$$
\begin{aligned}
& {\left[\begin{array}{r}
\mathbf{I}(0, s) \\
-\mathbf{I}(d, s)
\end{array}\right]=} \\
& \quad\left[\begin{array}{rr}
\mathbf{Y}_{a}(s)+\mathbf{Y}_{b}(s) & \mathbf{Y}_{a}(s)-\mathbf{Y}_{b}(s) \\
\mathbf{Y}_{a}(s)-\mathbf{Y}_{b}(s) & \mathbf{Y}_{a}(s)+\mathbf{Y}_{b}(s)
\end{array}\right]\left[\begin{array}{c}
\mathbf{V}(0, s) \\
\mathbf{V}(d, s)
\end{array}\right]
\end{aligned}
$$

We can derive asymptotic equivalent circuit models that satisfy the relations (13) and (14), but omit in this paper due to space limitation (refer to [17] for detail).

\section{Illustrative example}

At first, we investigate the distribution of poles in the complex plane. The parameters of interconnects are set as fol- 


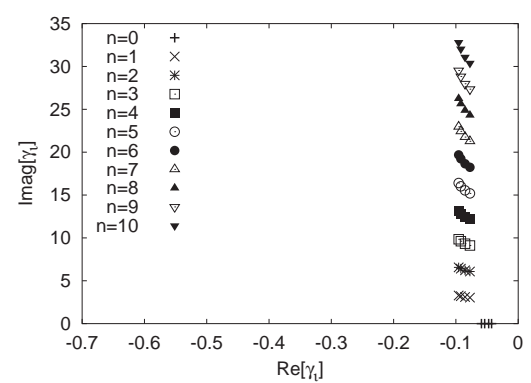

(a)

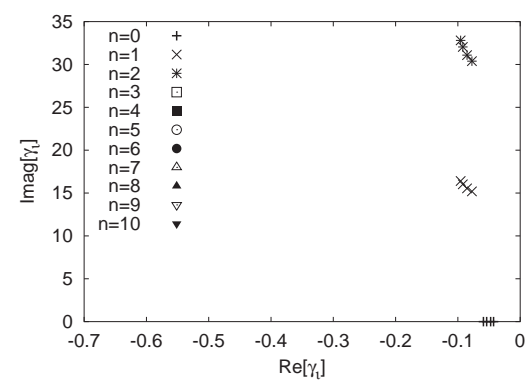

(b)

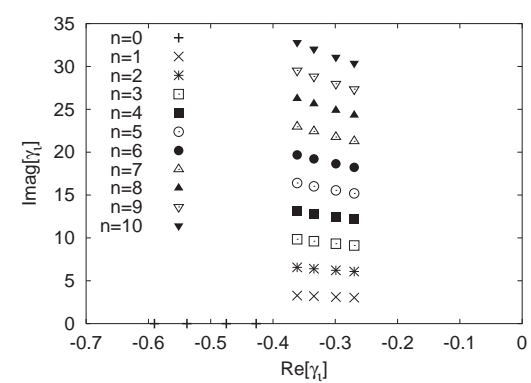

(c)

Fig. 1 Distribution of poles for 4-conductor interconnects. Poles with negative imaginary part are not included. (a) $r=0.5[\Omega / \mathrm{mm}], d=5.0[\mathrm{~mm}]$, (b) $r=0.5[\Omega / \mathrm{mm}], d=1.0[\mathrm{~mm}]$, (c) $r=5.0[\Omega / \mathrm{mm}], d=5.0[\mathrm{~mm}]$.

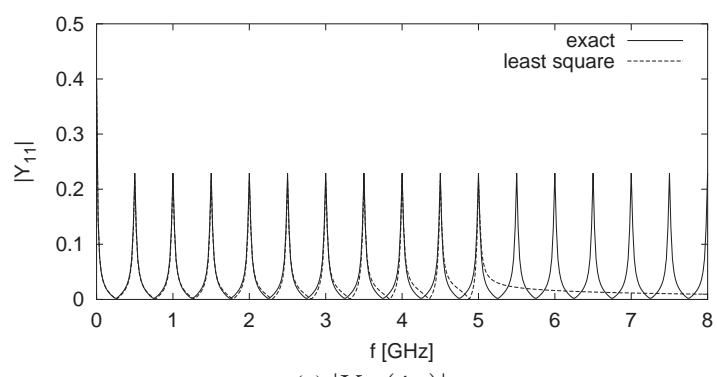

(a) $\left|Y_{11}(j \omega)\right|$

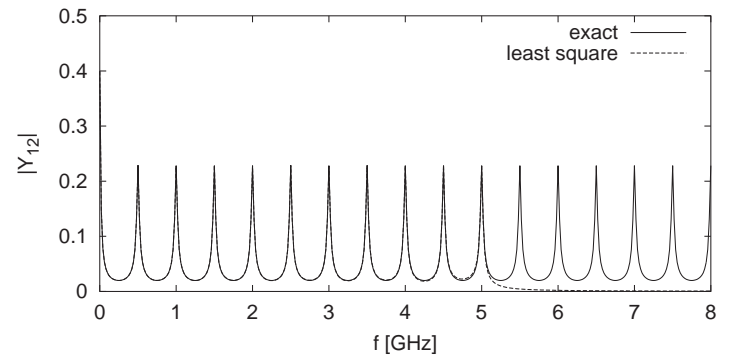

(b) $\left|Y_{12}(j \omega)\right|$

Fig. 2 Frequency response curves for single conductor interconnect. $M=10$

lows:

$R_{i, i}=r[\Omega / \mathrm{mm}]$, other elements of $\mathbf{R}$ are 0.

$L_{i, i}=10[\mathrm{nH} / \mathrm{mm}], L_{i, i+1}=L_{i-1, i}=1[\mathrm{nH} / \mathrm{mm}]$,

$L_{i, i+2}=L_{i-2, i}=0.1[\mathrm{nH} / \mathrm{mm}]$, other elements of $\mathbf{L}$ are 0 .

$C_{i, i}=4[\mathrm{pF} / \mathrm{mm}], C_{i, i+1}=C_{i-1, i}=-0.21[\mathrm{pF} / \mathrm{mm}]$,

$C_{i, i+2}=C_{i-2, i}=-0.01[\mathrm{pF} / \mathrm{mm}]$, other elements of $\mathbf{C}$ are 0 .

$G_{i, i}=0.5[\mathrm{mS} / \mathrm{mm}], G_{i, i+1}=G_{i-1, i}=-0.05[\mathrm{mS} / \mathrm{mm}]$,

$G_{i, i+2}=G_{i-2, i}=-0.01[\mathrm{mS} / \mathrm{mm}]$, other elements of $\mathbf{G}$ are 0 .

Fig. 1 shows the distribution of poles for 4-conductor interconnects. Observe that they consist of $N$ real poles and the complex conjugate poles for each $n$ in (5). As shown in Fig. 1 the imaginary parts of the complex conjugate poles increase monotonically as $n$ in (5). We found that the imaginary parts of the poles for short length interconnect become quickly large for $n$, Also the real parts of the poles become large, as the resistance component $r$ of interconnect increases. Therefore, we will need to take into account only a few dominant poles for either short-length interconnects or high-resistance one, such as ASIC, SoC and so on.

It is also found that the poles for $N=1$ always locate around the center position of pole distributions for another $N$. This means that the reduction technique for RCG interconnects [18] may be applicable to RLCG interconnects.

Using these exact poles, we calculated the residues by the least squares method. The frequency response curves approximated by substituting the obtained residues and poles to the partial fraction (13) and the exact curves obtained from (3) for single conductor interconnect are shown in Fig. 2. As found form this figure, the frequency response curves of the admittance matrices have many peaks correspond to the poles.
Both curves have relatively good agreement up to 11-th peak around $5[\mathrm{GHz}]$, because we took a real pole and 10 pairs of complex conjugate poles. For multi-conductor interconnects, we confirmed that the approximated curves are relatively consistent with the exact one, although due to the effects of coupling the shapes of the curves become more complicated than those of single conductor interconnect.

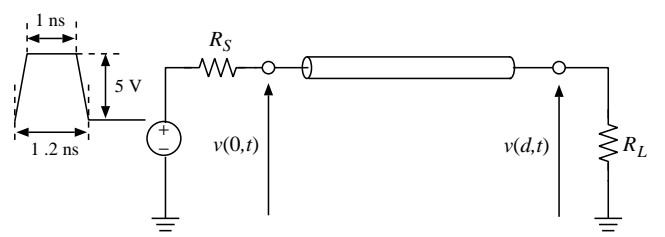

Fig. 3 Simple linear circuit with single conductor interconnect. $r=0.5[\Omega / \mathrm{mm}], d=5.0[\mathrm{~mm}], R_{S}=10[\Omega], R_{L}=10[\Omega]$

Next, by using the numerical inverse Laplace transformation [19], a simple linear circuit with a single conductor interconnect shown in Fig. 3 is simulated for both the exact admittance matrices (3) and the approximated partial fractions (13). The parameters of the method are $a=0.2, K=512$. Note that the numerical inverse Laplace transformation method can be applied to only linear circuits, but we use this method in order to investigate the effects of the reduction approximating the admittance matrices (3) with the partial fractions (13). Fig. 4 shows the transient response waveforms. We found that the more the number of poles increases, the more both waveforms are well in agreement.

\section{Conclusions and remarks}




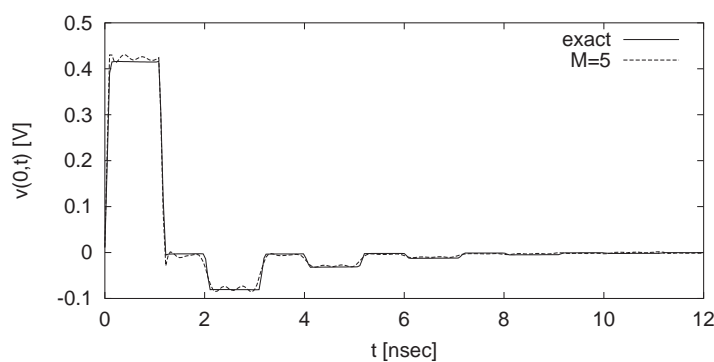

(a) near end. $M=5$

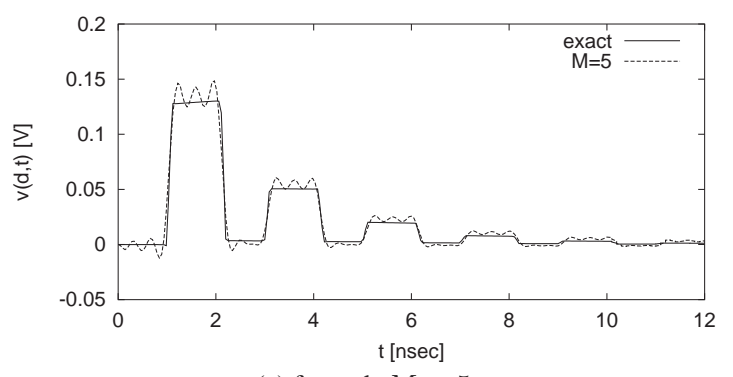

(c) far end. $M=5$

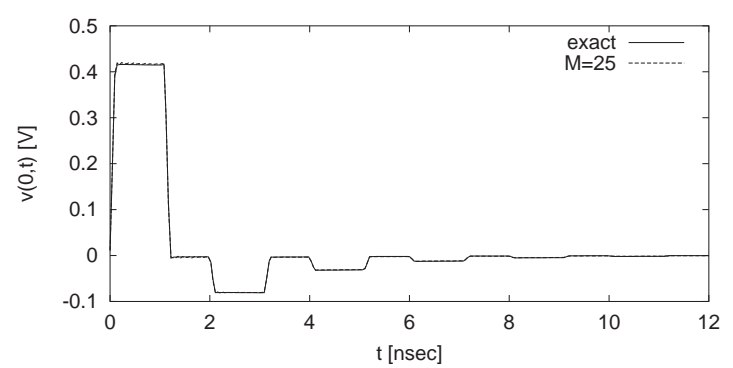

(b) near end. $M=25$

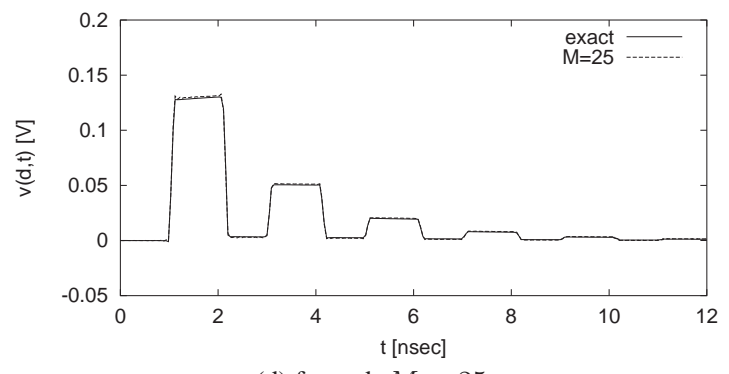

(d) far end. $M=25$

Fig. 4 Transient response for simple linear circuit of Fig. 3.

In this paper, we have proposed an algorithm for calculating the exact poles, the corresponding residues are estimated by the least squares method. Using the poles and residues, each element of the admittance matrices of interconnects is approximated by partial fraction.

In our simulations, it has been shown that the exact frequency response curves and the curves approximated by partial fractions have relatively good agreement. Further from transient simulation making use of the numerical inverse of Lapalace transforms, the response waveforms obtained by our method become more accurate, as the number $M$ of the complex conjugate pairs increases.

In future, we intend to find analytically the residues and compare the results obtained in this paper.

\section{References}

[1] J. A. Brandao Faria, Multiconductor Transmission-Line Structures: Modal Analysis Techniques, John Wiley and Sons, Inc., 1993.

[2] M. Celik, L. Pileggi and A. Odabasioglu, IC Interconnect Analysis, Kluwer Academic Pub., 2002.

[3] J. L. Wyatt, Jr., "Signal propagation delay in RC models for interconnect," Circuit Analysis, Simulation and Design, A. E. Ruehli(Editor), Elsevier Science Pub. B.V.(North-Holland), pp. 254-291, 1987.

[4] W. C. Elmore, "The transient response of damped linear networks with particular regard to wideband amplifiers," J. Appl. Phys., vol. 19, pp. 55-63, Jan. 1948.

[5] T. Sakurai, "Closed-form expressions for interconnection delay, coupling, and crosstalk in VLSI's," IEEE Trans. Electron Devices, vol. 40, pp. 118-124, 1991.

[6] S-Y. Kim, N. Gopal and L. T. Pillage, "Time-domain macromodels for VLSI interconnect analysis," IEEE Trans. Computer-Aided Design, vol. 13, pp. 1257-1270, 1994.
[7] R. Gupta, B. Tutuianu and L. T. Pileggi, "The Elmore delay as a bound for RC trees with generalized input signals," IEEE Trans. Computer-Aided Design, vol. 16, pp. 95-104, 1997.

[8] C. J. Alpert, A. Devgan and C. V. Kashap, "RC delay metrics for performance optimization," IEEE Trans. Computer-Aided Design, vol. 20, pp. 571-582, 2001.

[9] T. Kimura and M. Okumura, "An efficient reduction method of a substrate RC network model," IEICE Trans. Fundamentals, vol. E84-A, pp. 698-704, 2001.

[10] E. Chiprout and M. S. Nakhla, Asymptotic Waveform Evaluation and Moment Matching for Interconnect Analysis, Kluwer Academic Pub., 1994.

[11] E. Chiprout and M. S. Nakhla, " Analysis of interconnect networks using complex frequency hopping(CHF)," IEEE Trans. Computer Aided Design, vol. CAD-14, pp. 186-200, 1995.

[12] Q. Yu, J. Meiling and E. S. Kuh, "Passive multipoint moment matching model order reduction algorithm on multiport distributed interconnect networks," IEEE Trans. Circuits and Systems-I, vol. 46, pp. 140-160, 1999.

[13] P. Feldmann and R. W. Freund, "Efficient linear analysis by Padé approximation via the Lanczos process," IEEE Trans. Computer Aided Design, vol. CAD-14, pp. 639-649, 1995.

[14] A. Odabasioglu, M. Celik and L. T. Pileggi, "PRIMA: Passive reduced-order interconnect macromodeling algorithm," IEEE Trans. Computer Aided Design, vol. 17, pp. 645-654, 1998.

[15] A. Ushida, K. Urabe, Y. Yamagami and Y. Nishio, "Asymptotic equivalent circuits of interconnects based on complex frequency method," ECCTD'01, vol. 3, pp.29-32, 2001.

[16] D. K. Faddeeva and V. N. Faddeeva Computational Methods of Linear Algebra, W. H. Freeman and Co., 1963.

[17] J. Kawata, Y. Tanji, Y. Nishio and A. Ushida, "Asymptotic equivalent circuits of interconnects and the passivity," Tech. Rep. of IEICE, no.NLP2003-134, pp.43-48, 2003.

[18] Y. Yamagami, Y. Nishio, A. Hattori and A. Ushida, n"A reduction technique of large scale RCG interconnects in complex frequency domain," ISCAS'03, vol. 3, pp.490-493, 2003.

[19] A. Yonemoto, T. Hisakado, and K. Okumura, "An improvement of convergence of FFT-based numerical inversion of Laplace transforms", ISCAS'02, vol. V, pp. 769-772, 2002. 